\title{
Papers
}

\section{Process and impact of mergers of NHS trusts: multicentre case study and management cost analysis}

Naomi Fulop, Gerasimos Protopsaltis, Andrew Hutchings, Annette King, Pauline Allen, Charles Normand, Rhiannon Walters

\begin{abstract}
Objective To study the processes involved in and impact of mergers between NHS trusts, including the effect on management costs.

Design Cross sectional study involving in depth interviews and documentary analysis; case study to compare savings in management costs between case trusts and control trusts.

Setting Nine trusts (cross sectional study) and four trusts (case study) in London.

Participants 96 interviews with trust board members, other senior managers, clinicians, service managers, and representatives of health authorities, regional office, community health councils, local authorities, other trusts in the area, and primary care groups and trusts.
\end{abstract}

Main outcome measures Stated and unstated drivers, and impact of merger on delivery and development of services, management structures, and staff recruitment, retention, and morale. Effects of difference in trust size before and after the merger. Savings in management costs two years after merger. Results Some important drivers for merger are not publicly stated. Mergers had a negative effect on delivery of services because of a loss of managerial focus on services. Planned developments in services were delayed by at least 18 months. Trusts' larger sizes after mergers had unintended negative consequences, as well as predicted advantages. The tendency for one trust's management team to dominate over the other resulted in tension. No improvement in recruitment or retention of clinical and managerial staff was reported. Perceived differences in organisational culture were an important barrier to bringing together two or more organisations. Two years after merger, merged trusts had not achieved the objective of saving $£ 500000$ a year in management costs. Conclusions Important unintended consequences need to be accounted for when mergers are planned. Mergers can cause considerable disruptions to services, and require greater management support than previously acknowledged. Other organisations undergoing restructuring, such as primary care groups developing into primary care trusts and health authorities merging into strategic health authorities, should take these findings into account.

\section{Introduction}

Many mergers and reconfigurations of NHS trusts have taken place in recent years. Since 1997, 99 mergers of trusts have taken place; 14 in London (Department of Health, personal communication, 2001). These include horizontal mergers of acute hospitals, mental health trusts, and community health services trusts. More recently, primary care groups have merged to create primary care trusts. ${ }^{1}$ Reconfigurations have often been contentious politically-they even provided the focus in one constituency during the general election of 2001. ${ }^{2}$ This led to the establishment of the independent reconfiguration panel, which aims to adjudicate on proposals about mergers and reconfigurations to "take the politics out" of such decisions. ${ }^{3}$

There is a range of drivers for trust mergers. One aim is to achieve economic gains: firstly, by taking advantage of economies of scale and scope (especially with regard to management costs), ${ }^{4}$ and secondly, as a result of rationalising the provision of services by reducing excess capacity to treat patients. ${ }^{4}$ Some people assume that clinical quality improves as usage of specialised units increases, ${ }^{5-7}$ quality of medical training increases, ${ }^{8}$ and staff recruitment and staff retention become more effective. ${ }^{9}$

Political drivers for mergers include facilitating hospital or service closures, securing financial viability of smaller organisations, and (on the part of providers) ensuring increased negotiating power and a survival strategy by pooling resources and enlarging the organisation in response to challenges from purchasers of services. ${ }^{10}$ For mental health services, the belief that mental health trusts with a single focus can provide higher quality services has provided additional impetus for mergers. ${ }^{11} 12$

Sceptics of mergers argue that the evidence for benefits of horizontal mergers is patchy, contradictory, and often based on managers' beliefs about the benefits. ${ }^{40}$ Unintended consequences and potential drawbacks of mergers receive less attention. These include disruption of services as a direct consequence of mergers, diseconomies of scale, and problems with staffing, service integration, systems integration, and working practices, as well as issues of equity and access to services. $^{41013}$
Health Services
Research Unit,
London School of
Hygiene and
Tropical Medicine,
London
WC1E 7HT
Naomi Fulop
senior lecturer in
health service delivery
and organisational
research
Gerasimos
Protopsaltis
research fellow
Andrew Hutchings
lecturer in health
services research
Annette King
research fellow
Pauline Allen
lecturer in
organisational
research
Charles Normand
professor of health
economics
Rhiannon Walters
honorary research
fellow
Correspondence to:
N Fulop
naomi.fulop@
lshtm.ac.uk
bmj.com 2002;325:246 
We studied the processes involved in and impact of trust mergers. We performed a cross sectional study of the drivers and objectives of trust mergers, a management cost analysis, and an in depth, longitudinal, qualitative case study of four recent mergers in London.

\section{Methods}

We studied the process of merger in a cross sectional study of all nine trust mergers and reconfigurations in London which became established in April 1998 and April 1999. These involved the dissolution of 25 constituent trusts and the creation of 11 newly merged trusts. We also conducted in depth, longitudinal case studies of four of these mergers, established in April 1999.

\section{Cross sectional study}

The cross sectional study comprised an analysis of the public consultation documents for the nine proposed trust mergers and semi structured interviews with 14 managers in seven health authorities associated with the trusts. One future health authority refused to participate in the study. We aimed to identify the "stated" and "unstated" (not publicly stated) objectives of each merger.

\section{Case study}

In the case study, we studied one merger involving acute care trusts, one involving mental health trusts, and two involving community NHS trusts over a two year period in the second and third years after merger. These particular mergers were chosen to reflect the range of trust types and geographical areas. ${ }^{14}$ The case study aimed to explore the process of merger in depth, to assess how well the mergers' objectives had been met, and to determine the intended and unintended consequences of the mergers.

We report our findings from the first round of data collection, which took place in the second year after merger. During this stage, we interviewed a range of stakeholders from inside and outside the trusts. We interviewed 22-26 people per case (96 interviews in total). A core group of informants was interviewed in every case. From inside the trust, this included at least six board members. From outside the trust, this included

- Senior regional office representative

- Chief executive or chair of primary care group or primary care trust

- Director or assistant director of social services

- Chief officers of community health councils

- Chief executives of "sister" trusts.

Other informants were found through a snowballing technique (this entails identifying initial interviewees, who go on to recommend other people for interview).$^{15}$ We asked interviewees about the drivers for merger, the processes involved in the merger, and their assessment of the merger's impact on a range of issues related to service delivery, including objectives set before the merger.

Five members of the project team independently read the interview reports to ensure the analysis was reliable. We analysed data by creating a preliminary framework based on the theory behind the study and by organising responses around other themes that emerged from the data. ${ }^{16}{ }^{17}$ The project team discussed emergent themes and agreed on those to be included.

Savings in management costs for the four trusts in the case study were estimated by comparing the actual costs (as reported in audited annual accounts for the first two years after the merger) with an estimate of the costs if no merger had taken place. A control group of eight trusts not involved in a merger or major reconfiguration (any change in income $>20 \%$ in successive years) since 1995-6 was used to predict the costs if no merger had taken place. We treated 1997-8 as the base year, and we predicted management costs and income for the eight unmerged constituent trusts for 1999-2000 and 2000-1 on the basis of the control trusts' costs and income (as reported in their audited financial accounts). We used a sensitivity analysis to test the effect of different assumptions on the relation between income and management costs. The postmerger trusts used in the case study did not simply amalgamate the services of their constituent trustssubstantial increases and decreases in income associated with changes in provided services were seen. We adjusted the estimated savings in costs in 1999-2000 and 2000-1 to account for differences between actual and predicted incomes by using the different levels of variable costs produced by the sensitivity analysis.

\section{Results}

\section{Stated and unstated drivers}

Stated drivers (from public consultation documents) gave the official view of the background to and reasons for the merger, as well as the favoured organisational structure. These included a need to make internal savings in management costs and invest savings into services for patients, to safeguard specialist units and guarantee developments in services, to ensure that quality and amount of services provided were maintained in the light of external policy drivers that put additional pressures on services, eg Turnberg report, ${ }^{8}$ and to improve conditions and career prospects for staff and solve recruitment and staff retention problems. For acute trusts, there was a need to respond to various service and policy reviews that recommend concentration of some acute and specialist services. Community and mental health trusts were responding to beliefs that trusts' cooperation with local authorities would be better if coterminosity was achieved (that is, that the new trusts would cover the same geographical area as the local authority). Community trust mergers aimed to support development of primary care.

Unstated drivers were concerned with specific local issues about one or more of the constituent trusts and were as reported by key stakeholders during interviews. These included a need to impose new management regimes on trusts perceived by health authorities or regional office as "undermanaged" or "lacking control," to negotiate reductions in accumulated deficits of one of the constituent trusts (because new organisations could not be expected to carry the burden of deficit from the start), and to respond to lobbying from stakeholdersincluding central government, influential institutions, and pressure groups-on behalf of one or more constituent trusts. "The organisational merger was mixed up with the future of the [trust's] site, although 


\section{Box 1: Impact of merger on service delivery and development}

\section{Acute trust (merger of constituent trusts A and B)}

Negative aspects of merger

- Proposed changes to services in pathology, accident and emergency, and maternity departments still not implemented and delays continue, two years after the merger

- Most respondents from outside the trusts had looked forward to a fusion of the constituent trusts' strengths (of the experience of trust A's clinicians with trust B's management credentials); they were disappointed by the lack of progress, and they felt the merger had a negative effect on trust B's services and the morale of its staff

- "Policy changes regarding protocols and integrated care pathways are being implemented here-something that has been occurring in trust B for years. The nurses have said that this is what they want. It obviously takes time to filter through" (senior nurse, obstetrics and gynaecology)

- "It is difficult to transfer good practice because of the underlying distrust and prejudice toward the other [trust], and because people haven't themselves changed. There persists a difference between specialties and services. Clinicians talk of other clinicians (from the other trust) as if they are part of a different organisation and a unified organisational identity varies from service to service" (primary care group representative)

\section{Community trust I (merger of constituent trusts $\mathrm{C}$, D, and $\mathrm{E}^{*}$ )}

Negative aspects

- Impatience over delays in implementing actions after service reviews and an awareness of bureaucratic barriers to change in the larger, merged organisations are increasing. In community trust I, a proposed review of paediatric treatments had not been completed two years after the merger; this increased the review period to over 12 months

Positive aspects

- After initial resistance, the professional supervision and development policy introduced across the community trust was felt to enhance service delivery: "Now, for me as a manager [staff appraisal] helps me focus on certain areas, it's a useful process ... The staff appreciate it, most find it helpful. We set both team and personal objectives" (borough manager, community trust I)

\section{Community trust II (merger of constituent trusts F, G, and $\mathbf{H}^{*}$ )}

Negative aspects

- Delays in appointment of middle managers held up development of intermediate care services

Positive aspects

- The merger resulted in some sharing of good practices across constituent trusts: "There have been some positive things-there's been sharing of good practice. The staff might not support this, but some have been swapped about - our associate directors for this patch [a jobshare] came from [trust G] and brought different models-it's been good cross fertilisation" (primary care group representative)

\section{Mental health trust (brought together mental health services from four trusts: J, K, L, and $M^{*}$ )}

Negative aspects

- The process of merger distracts senior managers considerably from patient care: "The costs of restructuring are very hidden but very colossal. The negative impact of merging is that time and attention of senior people gets drawn away - on looking on policies, budgets, etc. We were disrupted by efforts to try and merge our information systems and find new headquarters. There is an incredible disruption to the patient focus agenda. You become more business oriented and it steers agenda away from patient care" (director of operations, mental health trust)

Positive aspects

- The merger achieved coterminosity with local authorities, which facilitated greater integration with social services: "The creation of directorates allowed for the focus on boroughs, it opened up the integration with social services, without any problems, and it allows us to become even larger" (executive board member, mental health trust)

- The merger facilitated the development of some smaller services: "[The merger] pulled together many experienced and good clinicians who previously did not have a dedicated management. They had been left to fight with adult mental health services for resources and for support. It brought together child and adolescent mental health services and helped us gain an identity as a service, with a stronger voice with purchasers and enabled the share of good working practices" (service manager, mental health trust)

- Merger has some benefits for service delivery: "The transfer of patients [across psychiatric intensive care units] is much easier now, as we are a larger organisation. This has taken pressure off hospital beds, and it has brought specialists together. We still do referrals, but the process is less complicated as it's mostly internally done" (senior manager, mental health trust)

*These mergers were complex reconfigurations whereby constituent trusts' services were amalgamated into more than one merged trust.

this went through a separate consultation process ... subsequently, there was a lot of negotiation around the conditions of the merger" (health authority interviewee involved in an acute trust merger).

\section{Impact of merger on service delivery}

The results from all four cases showed that the mergers had a negative effect on the delivery and development of services (box 1). Interviewees from inside and outside the trusts reported that the loss of managerial focus on services during the merger had some detrimental effects on patient care. Service developments were delayed by at least 18 months, and senior management had underestimated the timescale and effort involved in the mergers. Some positive effects of 
mergers on service developments were reported; these related, for example, to the fact that there would be more clinicians in smaller services to run them effectively (box 1).

\section{Trust size-does it matter?}

A range of advantages and disadvantages of large trust sizes was reported. Although the advantages were mostly stated objectives of the merger, the disadvantages were unintended consequences.

The benefits of larger organisations created by mergers include the presence of a larger pool of professional staff; this enables organisations to develop large teams of specialists, which allow clinical excellence to be achieved. Previously fragmented specialist services become unified and enhanced-for example, the child and adolescent mental health services in mental health trusts. Community trust managers thought local authorities paid more attention when trusts became larger. In the mental health trust, the increased size was thought to facilitate cross fertilisation of ideas. Increased opportunities for staff training were an immediate and tangible benefit, and professional networks were enhanced by the increase in the number of professionals.

The drawbacks included the fact that staff felt that senior managers had become remote, and service managers felt cut off from the services that they were managing. Staff in the acute trust felt that senior managers did not devote enough time to them and that their needs for help from the managers were ignored. Respondents originating from smaller trusts felt a loss of the informality and familiarity of the previous organisations and a decrease in the autonomy of services and local decision making. Large trusts were seen as unresponsive and slow to make decisions. External stakeholders were concerned about the ability of large trusts to oversee continued quality of services and patient care. Agencies used to having direct access to senior management had to deal mainly with middle management-this compromised strategic developments in some areas of service. The larger geographical area covered by the merged trusts increased travelling time between sites for managers. Internal communication was negatively affected-processes of communication were viewed as incoherent and slow in merged trusts

\section{Management structures}

Changes in the trusts' management structures created tensions within staff groups and between clinical staff and management. Although the competition for management posts followed NHS guidelines, the new senior management team tended to consist predominantly of staff from one of the constituent trusts; this created the impression of a "takeover" for many staff (box 2). On the positive side, mergers provided individual staff and services with an opportunity to emerge from the constraints of previously stagnating services and management organisations.

\section{Staff recruitment, retention, and morale}

Findings to date have not revealed a substantial improvement in staff recruitment or retention during the early stages of mergers, despite this being a prominent stated driver. Benefits to staff of mergers included improved systems of clinical supervision, more coherent professional management, and the advantages of programmes of appraisals, training, and career development that have been implemented. Clinical and managerial staff, however, emphasised the stress caused by the perceived imposed uncertainties and changes and the increase in workload associated with the process of merger.

\section{Organisational culture}

Respondents used the term "culture" to highlight the differences between the organisations and to explain conflicts of values and priorities. Differences in culture related to attitudes to innovation and risk taking-an outcome or process orientation-and patterns of communication (box 2).

Financial issues and management cost analysis

Interviews with finance managers indicated that the clearest source of potential savings from the merger was the $£ 500000-£ 750000$ that was associated with reduced numbers of members of management boards in the merged trusts. Finance managers were less convinced that other savings were achieved within the first financial year, and they had no clear evidence that savings were reinvested into services. Instead, they thought the mergers highlighted hidden financial problems in the constituent trusts and revealed differences in funding and staffing of services across the merged organisations.

Our cost analysis indicates that reductions in management costs after the mergers were less than the estimated target savings of $£ 500000$ a year. Regression analysis of the results from all 49 London trusts in 1997-8 (excluding teaching hospitals and single speciality trusts) indicated a strong linear relation, with the variable element of management costs estimated at $4.9 \%(95 \%$ confidence interval $4.5 \%$ to $5.4 \%)$ of income. On the basis of the actual variable costs in the control trusts, average management cost savings were calculated as $£ 178700$ (-£20 300 to $£ 377800$ ) in the first year after merger and $£ 346800$ ( $\$ 5300$ to $£ 688200$ ) in the second (see table). The savings were not consistent between the trusts in the case studies, and they were sensitive to changes in the variable cost assumptions used in the sensitivity analysis.

\section{Discussion}

The formation of large organisations through mergers had benefits and drawbacks. Benefits were mostly stated objectives of the mergers, but drawbacks arose during the process of merger and were not considered when the decision on whether to merge was made. When mergers go ahead, drawbacks-such as reduced accessibility for external stakeholders-need to be resolved by the people implementing the merger.

Some research has investigated whether mergers should take place (in terms of the optimal size of hospitals).$^{18}$ Much less research has investigated what happens after hospitals have merged, ${ }^{4}$ and even less has looked at the consequences of merging organisations that provide mental health or community health services. Argyris and Schon differentiated between an organisation's "espoused theory," which tends to be explicit, and an organisation's "theory in use" (actual pattern of activity), which tends to be tacit. ${ }^{19}$ They 


\section{Box 2: Reactions to mergers}

\section{New management structures}

Negative aspects

- "[Staff] appointment was a transparent process with external involvement, but it isn't perceived like this by some staff-and it is perception that often counts" (senior manager, community trust I)

- "We all want the best managers, and if they all just happen to be from [trust B], then so be it, but I'm sure that [the other] managers were as capable and committed" (community health council representative, acute trust)

- "The manager I worked with was made redundant. It's difficult to cope with that and I found it difficult not to allow resentment to spill over. It's a difficult period having to adjust to new styles of management when you knew that there were casualties. It wasn't a painless exercise" (manager, mental health trust)

- "It felt like a takeover. It felt that the new chief executive would surround herself with her people. They did not put their cards on the table, and there was no thought about the impact on staff. It felt like a death every time you went to trust $\mathrm{E}$ and someone hadn't got a job" (manager, community trust I)

- "A limbo period [existed] because of the protracted appointment procedure when people weren't sure who they were reporting to. Managers were in transitional roles and not sure of the level and scope of their authority-that caused uncertainty in managers, which was transferred to staff" (senior manager, community trust II)

- "The signs haven't changed outside buildings, and that doesn't help, and we even have internal documents with [trust F's name] on them instead of the new name and logo" (manager, community trust I)

Positive aspects

- "My role has changed. I spend less time on patient care and have a more consultative role-I sit on groups and, for example, I'm working with social services on a joint project, which will employ two people. Now I'm consulted on services for older people, I'm thought of as someone to be asked. I used to feel that my view didn't count, but now it does" (manager, community trust I)

- "For developing this service, it's good not to be isolated. I cover [one part] of the trust and have an opposite member in [the other] and a management team that covers the night nurses. We can talk through and share now-we're on the same wavelength" (service manager, community trust II)

\section{Staff recruitment, retention, and morale} Negative aspects

- "There is a $60 \%$ vacancy rate in school nurses, and that's because of the merger. The merger delayed a review of roles and job descriptions, so we've fallen behind others. The review is starting now, but we've got to bring in a consultant to do it. That's directly because of the merger, and it's only one example. For the therapies, it helps to have more-there are now ten speech and language therapists and two dieticians in the trust (rather than one in each); it's nicer for them" (manager, community trust I)

- "We are short staffed, as people move on, and we have learnt to live with what's left. Their leaving is undoubtedly caused by the merger-they feel no security and no career pathway. Basically, no one wanted to move to [trust A]" (clinician, accident and emergency department, acute trust)

- "Huge pressure on existing staff; people I respect almost went under-they were reduced to their knees, taking on the extra workload and adjusting to the new organisation" (community health council representative, community trust II)

\section{Positive aspects}

- "There have been some benefits from merger on the periphery of training and development. [Trust E] had had an audit, which revealed that they were seeking better management. Appraisal, personal development plans, better supervision have all come to pass" (executive board member, community trust I)

\section{Differences in organisational culture between merging trusts}

- "There might be four miles difference between us, but there is two decades in terms of culture and practice" (executive board member, acute trust)

- "It was hard to deal with two systems, cultures, working practices, etc. It was difficult to get people to change their working practices and that took a lot of time" (local authority representative, acute trust)

- "The cultures are different and it's very difficult to get people to change their culture. We wouldn't like someone coming in and telling us what we've done up till now is wrong" (manager, acute trust)

- "[Community trust I] seems very process oriented, and that's a culture that's come from [trust C]. You need processes, but you also need creativity. [Trust E] was low on processes, which was a weakness, but high on innovation and creativity. It had a flat management structure. It was an organisation prepared to support risk, and to support failure when things go wrong. [Trust C] tend to blame the individual. I championed an innovative nurse led service, which is not championed now-and it's falling apart. [Trust C] culture dominates and is more negative about innovation" (borough director, community trust I)

- "So you have one part of the trust being about staff development but not getting anything done, and another part quite decisive and accountable but with a bit of a spirit of cultural deprivation" (executive board member, mental health trust)

argued that low congruence between the two largely accounts for the organisation's identity.

We analysed two types of drivers for mergers: "stated" drivers-as set out in the formal consultation documents ("espoused theory")-and "unstated" drivers-revealed through interviews with trust and health authority staff ("theories in use"). Both types of drivers are important to understand, as they provide the context for the merger process-particularly where "unstated" drivers conflict with "stated" drivers (which 
Estimated savings in management costs in the study trusts in the first two years after merger, by cost assumption (\% of income) used in sensitivity analysis

\begin{tabular}{|c|c|c|c|c|c|}
\hline Variable & \multicolumn{5}{|c|}{ Savings (£000) } \\
\hline \multicolumn{6}{|c|}{ First year after merger (1999-2000) } \\
\hline $\begin{array}{l}\text { Cost assumption (\% of } \\
\text { income) }\end{array}$ & 5.15 & 5.00 & $4.85 \dagger$ & 4.70 & 4.55 \\
\hline $\begin{array}{l}\text { Mean saving in } \\
\text { management costs } \\
(95 \% \mathrm{CI}) \ddagger\end{array}$ & 59.8 (-104.5 to 224.1) & 119.3 (-55.6 to 294.2$)$ & $178.7(-20.3$ to 337.8$)$ & $238.2(-5.6$ to 470.8$)$ & 297.5 (25.6 to 569.5$)$ \\
\hline \multicolumn{6}{|l|}{ Savings in trust: } \\
\hline 1 & 76.7 & 34.5 & -7.6 & -49.7 & -91.7 \\
\hline 2 & 80.8 & 169.6 & 258.5 & 347.4 & 436.1 \\
\hline 3 & -79.7 & -4.3 & 71.2 & 146.7 & 222.0 \\
\hline 4 & 161.5 & 277.2 & 392.8 & 508.5 & 623.8 \\
\hline \multicolumn{6}{|c|}{ Second year after merger (2000-1) } \\
\hline $\begin{array}{l}\text { Cost assumption (\% of } \\
\text { income) }\end{array}$ & 4.45 & 4.3 & $4.15 \dagger$ & 4.0 & 3.85 \\
\hline $\begin{array}{l}\text { Mean saving in } \\
\text { management costs } \\
(95 \% \mathrm{Cl}) \ddagger\end{array}$ & $\begin{array}{c}200.4(-129.6 \text { to } \\
530.4)\end{array}$ & $273.7(-56.8$ to 604.3$)$ & 346.8 (5.3 to 688.2) & 420.0 (58.1 to 781.9$)$ & 493.2 (102.8 to 883.5$)$ \\
\hline \multicolumn{6}{|l|}{ Savings in trust: } \\
\hline 1 & 273.8 & 230.4 & 187.1 & 143.8 & 100.5 \\
\hline 2 & 19.1 & 128.5 & 237.5 & 346.6 & 455.8 \\
\hline 3 & 198.8 & 293.0 & 386.8 & 480.7 & 574.7 \\
\hline 4 & 309.9 & 443.1 & 575.8 & 708.7 & 841.7 \\
\hline
\end{tabular}

$\dagger$ Actual variable costs for control trusts for relevant year.

fCompared with control trusts.

may leave the organisation unclear about its objectives). The "unstated" objectives highlight the political context within which mergers take place. Whether the independent reconfiguration panel will be able to "take the politics out of" these decisions remains to be seen.

\section{Restructuring}

For all four cases, the merger brought about a period of organisational restructuring and intensive introspection that set them back in terms of developments in the organisation and services by at least 18 months. The amount of time needed for restructuring was underestimated by the people who initiated the mergers and by those who implemented them. Such delays are important unintended consequences of mergers that must be taken into account in future plans for reorganisation. ${ }^{13}$

\section{Cultural differences}

Differences in cultures between merging organisations seem to be an important barrier to bringing organisations together through a merger. Particular aspects of organisational culture cited in our study-such as attitudes to innovation and risk taking and whether an organisation has more of an outcomes or process orientation, and patterns of communication-are highlighted as keys to the future direction of health services. ${ }^{20}$

\section{Trust size}

Larger organisations were expected to be able to provide better opportunities and facilities for staff, and this was expected to solve problems with recruiting and retaining staff. Our study shows that the merger had no positive impact on the recruitment and retention of clinical staff. In some cases, the mergers exacerbated the problems of recruiting and keeping managerial staff; they often left to avoid organisational disruption.

\section{Management costs}

Average savings in management costs of $£ 178700$ in the first year and $£ 346800$ in the second year after merger did not meet the clearly stated objective of reducing management costs by $£ 500000$ a year. These numbers should be treated with caution because of the small sample size and sensitivity of the analysis to assumptions about the relation between management costs and income, but our data support predictions that reductions in management costs after mergers would be small. ${ }^{21}$ The smaller calculated savings can be explained in part by the use of a control group of trusts in the analysis. Between 1997-8 and 2000-1, management costs in the control trusts increased by $3.6 \%$ and income increased by $26.7 \%$. We adjusted the estimated savings for the trusts used in the case studies to account for this general decline in management costs (as a proportion of income) reported by trusts not involved in mergers.

The low savings in management costs achieved, particularly in the first year after the merger, suggest that the implementation of mergers needed more management support than had been anticipated. Merged organisations thus need to set realistic objectives in terms of savings in management costs by taking into account the amount of managerial input needed to implement the merger. The possibility of cost savings and changes in efficiency in areas other than management costs was not explored, because this was not a stated objective of the mergers and because comparative cost data for all of the different types of trusts included in the analysis are lacking.

\section{Timing of study}

We analysed the drivers and consequences of merging healthcare organisations for the two years after merger. Our study was commissioned after the mergers had formally taken place. Ideally, research on mergers and reconfigurations should begin before the mergers take place. This would be very challenging to achieve in practice, however, as it could be argued that the process of merger begins as soon as discussions about merger begin. Findings presented in this paper are "interim," in 
the sense that they provide a picture of the impact of mergers at a relatively early stage in the life of the new organisation. Other studies in the United States and the United Kingdom showed that the results of mergers are disappointing and that it takes a long time for positive results to show. ${ }^{13} 2122$ Our study may have been performed too soon after mergers to judge whether or not they met their objectives, given the length of time taken to achieve considerable change in healthcare organisations. The results from the second stage of our data collection for the case study, which took place during the organisations' third year of operation (data are currently being analysed), may show that the merged trusts are closer to meeting the mergers' objectives. The longer the timeframe used, however, the more difficult it is to attribute effects-for example, the impact on service developments-to the merger process, given the context of a turbulent environment of change within the NHS.

\section{Conclusions}

It is important to understand the unintended consequences of merger and to set realistic objectives for these reorganisations. Our findings have implications for other NHS organisations that are experiencing reorganisation-such as the development of primary care groups into primary care trusts and the merger of health authorities into strategic health authorities. ${ }^{23}$

We thank the staff of the NHS and allied organisations that took part in this study and Trevor Sheldon for his comments on a previous version of this paper.

Contributors: NJF, AK, PA, and CN developed the protocol and together with AH obtained funding for the study. GP, AK, RW, NJF, AH, and CN conducted the interviews for the cross sectional study and the case study. GP and AK analysed the consultation documents. AK, GP, NJF, PA, and RW read the interview reports and agreed on the themes to be analysed. GP and AK conducted the full analysis of the interview data. $\mathrm{AH}$ and $\mathrm{CN}$ developed the method for analysing the management cost data. AH conducted the analysis. NJF, GP, and AH wrote the paper. PA, AK, CN, and RW commented on drafts of the paper. $\mathrm{NJF}$ is guarantor.

Funding: NHS Executive London Region's organisation and management research and development programme.

Competing interests: None declared.

1 Bojke C, Gravelle H, Wilkin D. Is bigger better for primary care groups and trusts? BMJ 2001;322:599-602.

2 Gulland A. Doctor wins seat in fight to save hospital. BMJ 2001;322:1443.

3 The NHS Plan. A plan for investment. A plan for reform. London: Stationery Office, 2000.

4 Ferguson B, Goddard M. The case for and against mergers. In: Ferguson B, Sheldon T, Posnett J, eds. Concentration and choice in healthcare. London: Royal Society of Medicine, 1997:67-82.

5 NHS Centre for Reviews and Dissemination. Concentration and choice in the provision of hospital services. The relationship between hospital volume and quality of health outcomes. University of York: Centre for Reviews and Dissemination, 1997. (CRD report No 8, part I.)

6 Hospital volume and health care outcomes, costs and patient access Effective Health Care 1996;2(8). www.york.ac.uk/inst/crd/ehc28.htm (accessed 20 June 2001).

\section{What is already known on this topic}

Research suggests that effectiveness increases as the amount of activity by specialised units in certain clinical specialities increases

Little empirical research has looked at the impact of mergers; most studies focus on financial variables

Mergers result in short term disruption caused by difficulties in integrating services and personnel

\section{What this study adds}

Important drivers for NHS mergers that are not stated publicly have implications for the process and impact of mergers

Mergers have positive effects, as well as unintended negative consequences that disrupt services and set back developments in services

Perceived differences in organisational culture impede bringing organisations together

Mergers do not achieve target savings in management costs in first two years after merger

7 Smith R. How to best organise acute hospital services? BMJ 2001;323:245-6

8 Dowie R, Gravelle H. Changes in medical training and sub-specialisation: implications for service delivery. In: Ferguson B, Sheldon T, Posnett J, eds. Concentration and choice in healthcare. London: Royal Society of Medicine, 1997:51-65.

9 Ferguson B, Sheldon T, Posnett J. Introduction. In: Concentration and choice in healthcare. London: Roval Society of Medicine, 1997:1-7.

10 Garside P. Evidence based mergers? BMJ 1999;318:345-6.

11 Department of Health. Strategic review of London's health services. Report of the independent advisory panel. London: Stationery Office, 1997.

12 Department of Health. Review of London health services. Government response to London review report. London: Stationery Office, 1998.

13 McClenahan J. Emerging problems with merger policy. In: Appleby J, Harrison A, eds. Health care UK 1999/2000: the King's Fund review of health policy. London: King's Fund, 1999: 94-7.

14 Bowling A. Research methods in health:investigating health and health services. Buckingham: Open University Press, 1997.

15 Burgess RG. In the field: an introduction to field research. London: Allen and Unwin, 1984.

16 Miles MD. Qualitative data as an attractive nuisance-the problem of analysis. Admin Sci Q 1979;24:590-601.

17 Bryman A, Burgess RG, eds. Analysing qualitative data. London: Routledge, 1994

18 Posnett J. Is bigger better? Concentration in the provision of secondary care. BMJ 1999; 319:1063-5.

19 Argyris C, Schon DA. Organisational learning II: theory, method and practice. Wokingham: Addison-Wesley, 1996.

20 Davies HT, Nutley SM. Organisational culture and quality of health care. Oual Health Care 2000;9:111-9.

21 Goddard M, Ferguson B. Mergers in the NHS: made in heaven or marriages of convenience. London: Nuffield, 1997.

22 Weil TP. Horizontal mergers in the United States health field: some practical realities. Health Serv Manage Res 2000;13:137-51.

23 Department of Health. Shifting the balance of power within the NHS securing delivery. London: Stationery Office, 2001.

(Accepted 28 February 2002) 